\title{
Descripción del proceso de la elaboración de tintes naturales y artificiales - Chincheros Cusco
}

\author{
Description of the elaboration process of natural dyes and artificial - Chincheros \\ Cusco
}

\author{
${ }^{1}$ Andrea Quenta Cabrera \\ ${ }^{2}$ Arturo Toledo Gonza
}

\begin{abstract}
RESUMEN
El presente artículo detalla la metodología para cumplir con los objetivos propuestos en la investigación. Los cuales ayudaron a identificar saberes ancestrales sobre el uso de plantas, para conocer y describir el proceso de elaboración de tintes naturales y artificiales en la comunidad de Chincheros (Cusco), que aún conserva en su memoria la sabiduría ancestral sobre las plantas y técnicas tintóreas. La pérdida cultural se debe, sobre todo, a la modernización y la no valorización de los conocimientos tradicionales; por tanto, si no se registran adecuadamente, corren el riesgo de perderse y extinguirse de la identidad étnica. El estudio reconstruye las técnicas tintóreas tradicionales usadas en la comunidad Andina del Perú, para su preservación y uso en la artesanía e industria textil contemporánea. La metodología propuesta incluye la evaluación de plantas con potencial tintóreo al igual que el uso de tintes artificiales, observando las cualidades de ambos. El proceso de teñido consta de cuatro fases ejecutadas de manera consecutiva. Como paso previo a la obtención del tinte, la preparación de la fibra es fundamental en el acabado del producto. Se experimentó con especies vegetales, de lo cual se obtuvieron diversas gamas de colores, extraídos de las plantas utilizadas por los pobladores de la comunidad Warmi Maki. Se concluye que el uso de plantas tintóreas en comunidades andinas aún se conserva en el contexto de su cultura tradicional.

Palabras clave: Comunidades, etnobotánica, plantas tintóreas.
\end{abstract}

ABSTRACT
This article details the methodology to meet the objectives proposed in the research. These helped to identify ancestral knowledge about the use of plants, to know and describe the process of elaboration of natural and artificial dyes in the community of Chincheros (Cusco), which still keeps in its memory the ancestral wisdom about plants and dyeing techniques. Cultural loss is mainly due to modernization and the failure to value traditional knowledge; therefore, if not properly recorded, it risks being lost and extinguished from the ethnic identity. The study reconstructs the traditional dyeing techniques used in the Andean community of Peru, for their preservation and use in contemporary crafts and the textile industry. The proposed methodology includes the evaluation of plants with dyeing potential as well as the use of artificial dyes, observing the qualities of both. The dyeing process consists of four phases executed consecutively. As a previous step to obtaining the dye, the preparation of the fibre is fundamental in the finishing of the product. Experiments were made with vegetable species, from which various ranges of colours were obtained, extracted from the plants used by the inhabitants of the Warmi Maki community. It is concluded that the use of dye plants in Andean communities is still preserved in the context of their traditional culture.

Keywords: Communities, ethnobotany, dye plants.

${ }^{1}$ Estudiante. Escuela Profesional de Arte. Facultad de Ingeniería Civil, Arquitectura y Geotecnia. Universidad Nacional Jorge Basadre Grohmann. E-mail: arte28quenta@gmail.com

${ }^{2}$ Docente. Escuela Profesional de Arte. Facultad de Ingeniería Civil, Arquitectura y Geotecnia. Universidad Nacional Jorge Basadre Grohmann. E-mail: atoledog@unjbg.edu.pe 


\section{INTRODUCCIÓN}

Esta investigación busca conocer y revalorizar antiguas técnicas de teñido artesanal, adaptándolas a nuestra época, ya que se encuentran a nuestro alcance, y así lograr una mayor difusión y aplicación de las técnicas de teñido artesanal en la actualidad.

El conocimiento sobre el uso de plantas tintóreas en el Perú es de larga trayectoria histórica, en especial en los aspectos vinculados a su uso en el teñido de textiles. Las culturas pre-incas son reconocidas por sus textiles y técnicas usadas para el teñido de fibras a partir del uso de recursos naturales. Estos conocimientos transgeneracionales fueron transmitidos horizontalmente (Zumbuhl, 1979). Debido a que fueron materiales perecibles, resulta difícil encontrar evidencias arqueológicas que permitan identificar las especies empleadas (Ivic y Berger, 2008). En la actualidad, a pesar del descubrimiento de los colorantes sintéticos (Sing, 1997), aún se conservan las técnicas empleadas desde la antigüedad, asociadas al uso de plantas tintóreas.

Los tintes naturales son de mayor importancia, esto debido a que pueden usarse en el teñido de hilo de algodón y lana, al igual que los tintes artificiales; sin embargo, comparando ambos tintes, tienen más relevancia los naturales en las comunidades encargadas del teñido.

Se puede destacar la forma en que los artesanos tintóreos han manejado durante milenios los recursos naturales para la obtención de colorantes, algunos de frágil estabilidad, cuidando de no agotarlos o bien recurriendo a su cultivo o domesticación, hasta la actualidad. (Jaramillo, 1988).

En la Comunidad Chincheros, departamento del Cusco, se utilizan estos tintes como herramienta de trabajo en el proceso de elaboración del teñido, siendo un lugar de demanda textil (Boucherie, 2014) $\mathrm{y}$ acogida internacional por turistas que visitan esta comunidad de artesanos que han rescatado la tradición del arte textil de nuestra historia.

"El estudio de la cultura y la discusión sobre sus representaciones y alcances, data de mucho tiempo atrás y ha sido preocupación primordial de varias corrientes antropológicas y sociales". (Korovkin, 2002, p.18).
El proceso químico para la obtención de colorantes, en ocasiones, provoca que se realicen diversos procedimientos con el objeto de conseguir productos colorantes con cualidades apropiadas a los fines textiles. Por ejemplo, los tintes artificiales necesitan de aglutinantes para su fijación y ser insolubles en agua. Por su reducción en un medio alcalino, se transforman en leuco derivados hidrosolubles (Crespi, 1987). Los químicos afectan la salud humana, en especial a la reproducción y perjudican el desarrollo de los niños, ya que dañan el hígado, el sistema inmunológico, etc. (Quintriqueo, Gutiérrez y Contreras, 2012).

\section{Objetivos}

Conocer y describir el proceso de la elaboración de tintes naturales y artificiales-Chincheros, Cusco.

\section{Hipótesis}

El estudio del proceso de elaboración de tintes naturales y tintes artificiales-Chincheros, Cusco permitirá conocer los beneficios y cualidades de los tintes.

\section{MATERIALES Y MÉTODO}

\section{Área de estudio}

Se realizó la recolección de información basada en el proceso del teñido y la obtención de tintes naturales en el departamento de Cusco, distrito de Chincheros, en la comunidad de artesanos llamada Warmi Maki (figura 1), donde se realizó la encuesta a 30 mujeres especialistas en el tema.

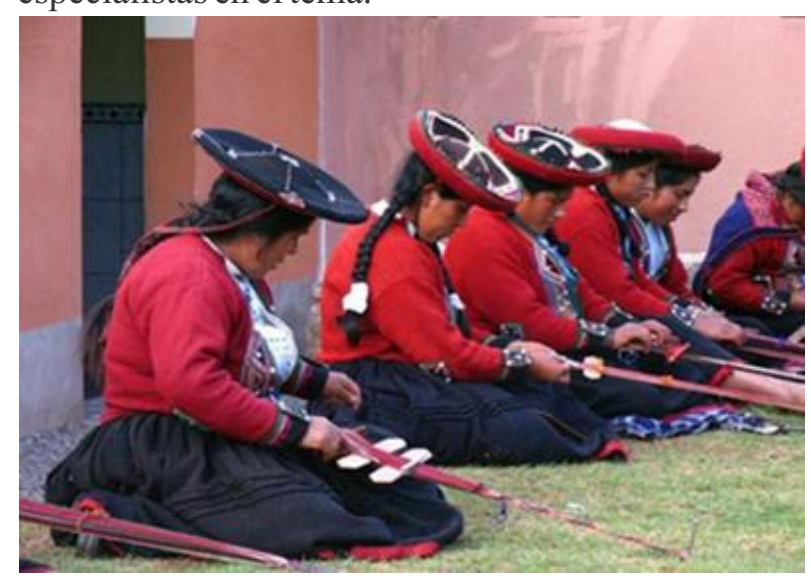

Figura 1. Tejedoras de Chincheros

Fuente: Andrea Quenta Cabrera 


\section{Metodología}

La presente investigación es de tipo descriptiva porque describe el proceso de elaboración de tintes naturales y artificiales de carácter cualitativo. Se utiliza la recolección de datos sin medición numérica para responder y afirmar preguntas de investigación, y se pueda o no probar la hipótesis en el proceso de interpretación. Se utilizó como técnica, la encuesta; la guía de entrevista y el cuestionario como instrumentos para determinar cuántas personas practican todavía el tinturado natural al igual que el artificial (anilinas), para lo cual se utilizaron fibras de origen animal (lana de oveja) y extracción de tintes vegetales y respectivos mordientes, fijadores según la entrevista. Se realiza el conocimiento de la realidad mediante la observación directa de las manifestaciones y expresiones socio cultural.

\begin{tabular}{ccccc} 
Comunidad & Distrito & Provincia & Región & $\begin{array}{c}N^{\circ} \text { De mujeres } \\
\text { encuestadas }\end{array}$ \\
\hline $\begin{array}{c}\text { Asociación de } \\
\text { Artesanas } \\
\text { Warmi Maqui }\end{array}$ & Chincheros & Urubamba & Cusco & 30 \\
\hline
\end{tabular}

Nota: Elaborado para conocer el número de muestra en el mes de enero de 2019

\section{RESULTADOS}

Para esta investigación, se propuso difundir los procesos del teñido artesanal a través de un taller de elaboración de tintes naturales y artificiales. Para la realización de este taller, el enfoque fue difundir los procesos del teñido de fibra natural (lana de oveja) con tintes naturales en nuevos escenarios donde se vincule la comunidad. Por ello, las mujeres expertas en este saber fueron quienes capacitaron a sus compañeros de la comunidad.

\section{El uso de mordientes}

El principio de la aplicación se denomina premordentado, ocurre cuando la lana se somete al mordiente antes de la tinción; y post-mordentado, cuando este se ejecuta luego de realizarse el teñido. El pre-mordentado facilita la captación y fijación de los colorantes disueltos. Por ejemplo, para $500 \mathrm{~g}$ de lana de oveja se requiere aproximadamente $50 \mathrm{~g}$ de alumbre (KAl (SO4)212H2O) previamente triturada. Luego, se vierte en la olla con agua caliente la lana de oveja lavada en el alumbre y se deja hervir por 40 minutos. Finalmente, se escurre y se lava la lana con abundante agua. Se deja reposar la lana hasta el momento del teñido. El Post-mordentado tiene como objetivo variar el color adquirido durante la tinción y reforzar la solidez al lavado y a la exposición a la luz (Cedano, 1994).

\section{Tinción}

Esta etapa consiste en la extracción del tinte de las plantas sometidas a una trituración o machacado, con el fin de romper tejidos vegetales y liberar los pigmentos, el tiempo de reposo varía según la especie. Se utiliza una amplia gama de tintes y procesos, según el tejido y acabado que se persigue.

\section{Penetración del colorante en la fibra de lana de oveja}

La fibra fue debidamente lavada con una raíz oriunda llamada saqta, la cual funciona como detergente y elimina residuos de grasa.

Luego que la fibra fue lavada y está completamente seca, se procede a colocarla en una olla con el tinte extraído de la planta. El proceso de maceración y cocción demora entre 40 a 45 minutos y el secado un día entero.

\section{Enjuague y secado}

En este paso, el propósito es que se enjuague por segunda vez con agua limpia, para que se adhiera el color. Se deja secar en la sombra, esto le brinda brillo y suavidad (Cornejo, 1987).

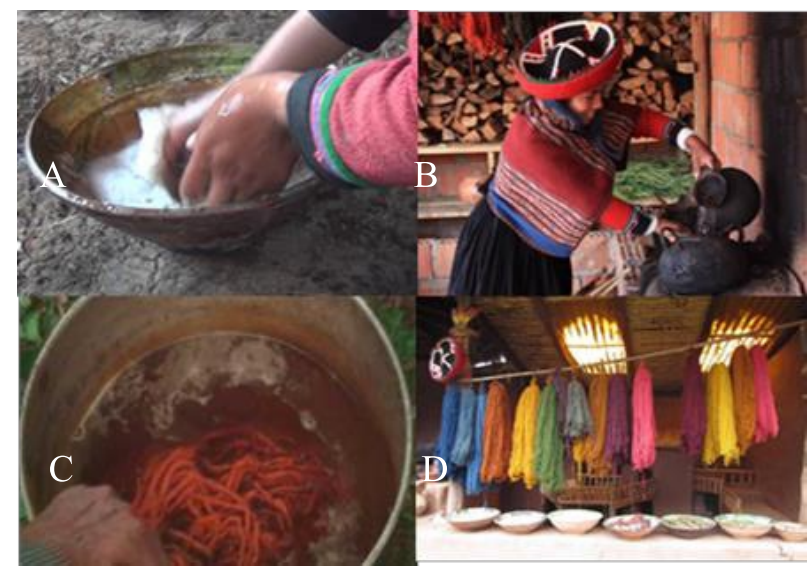

Figura 2. A. Lavado de lana de oveja. B. proceso de hervor del tinte. $C$. mezcla de la lana y tinte. $D$. secado de la lana teñida 


\section{Plantas utilizadas en la tinción}

De las plantas usadas para la extracción del tinte (tabla 2) se encuentra los colores más representativos como: verdes, rojos y amarillos.

Tabla 2. Plantas y colores extraídos

\begin{tabular}{ll}
\hline PLANTA & COLORES EXTRAIDOS \\
\hline Nogal (Juglans regia) & Marrón \\
Sauce Ilorón (Salix babilónica) & $\begin{array}{l}\text { Marrón claro, Marrón } \\
\text { amarillento } \\
\text { Rojo,carmín, naranja, }\end{array}$ \\
$\begin{array}{l}\text { alizo } \\
\text { Ginkgo (Ginkgo biloba L) }\end{array}$ & Amarillo claro, Verde, gris \\
$\begin{array}{l}\text { Tilo (Tilia platyphyllos) } \\
\text { Acacia (Robinia pseudoacacia }\end{array}$ & Morado, ladrillo \\
'Umbraculifera') & Amarillo, Marrón claro \\
$\begin{array}{l}\text { Palmera datilera (Phoenix } \\
\text { dactylifera) }\end{array}$ & Morado \\
Cedrón (Aloysia triphylla) & Marrón amarillento, Amarillo \\
$\begin{array}{l}\text { Granada (Punica granatum L.) } \\
\text { Helecho plumoso (Asparagus } \\
\text { plumosus) }\end{array}$ & Verde amarillento, Amarillo \\
$\begin{array}{l}\text { Helecho serrucho } \\
\text { (Nephrolepis exaltata) }\end{array}$ & Verde, Verde amarillento \\
\hline
\end{tabular}

Nota: Elaborado en base a los colores extraídos de plantas andinas

En la Tabla 3, se muestra que el $57 \%$ de mujeres artesanas consideran que el fijado del tinte natural es eficiente y el $33 \%$ afirma que los colores son más intensos y variados. Por último, el $10 \%$ afirma que estos tintes presentan una mejor duración. Por tanto, se puede concluir que las cualidades de los tintes naturales son más efectivos para los artesanos.

Tabla 3. Cualidades de los tintes naturales

\begin{tabular}{ccc}
\hline Tintes naturales & Frecuencia & Porcentaje \\
\hline Fijado rápido & 17 & $57 \%$ \\
intenso & 10 & $33 \%$ \\
Duración & 3 & $10 \%$ \\
total & 30 & $100 \%$
\end{tabular}

Fuente: Elaborado en base a la encuesta aplicada en el mes de enero de 2019
En la Tabla 4, el $67 \%$ de mujeres artesanas confirma que el fijado es inmediato. Asimismo, el $33 \%$ de los encuestados afirma que la intensidad del teñido es medianamente buena. Por último, se observa que ningún participante confirma que la duración del teñido pierde los colores con mucha facilidad. Por lo tanto, se afirma que las cualidades de los tintes artificiales no son efectivas para los artesanos.

Tabla 4. Cualidades de los tintes artificiales

\begin{tabular}{ccc} 
Tintes artificiales & frecuencia & porcentaje \\
\hline Fijado rápido & 20 & $67 \%$ \\
intensidad & 10 & $33 \%$ \\
duración & 0 & $0 \%$ \\
total & 30 & $100 \%$
\end{tabular}

Fuente: Elaborado en base a la encuesta aplicada en el mes de enero de 2019

La Tabla 5, muestra que el $33 \%$ de mujeres artesanas considera que la calidad de los tintes naturales es efectiva. Asimismo, el $30 \%$ afirma que la intensidad es adecuada respecto a los colores extraídos. Por último, el $37 \%$ sostiene que sus tonalidades son más variadas y de mejor calidad.

Tabla 5. Beneficios de los tintes naturales a diferencia de los tintes artificiales

\begin{tabular}{ccc}
\hline Tintes naturales & Frecuencia & Porcentaje \\
\hline calidad & 10 & $33 \%$ \\
intensidad & 9 & $30 \%$ \\
tonalidades & 11 & $37 \%$ \\
Total & 30 & $100 \%$ \\
\hline
\end{tabular}

Fuente: Elaborado en base a la encuesta aplicada en el mes de enero de 2019

\section{DISCUSIÓN}

Los pobladores del distrito de Chincheros tienen una larga tradición artesanal, textil y comercial. Dentro de estas tradiciones de carácter histórico, el tinturado natural es un conocimiento tradicional andino que desde los tiempos remotos ha sido utilizado, recreado e innovado desde las culturas prehispánicas, (Chincheros, 2011).

Algunos tintes extraídos de plantas requieren de un mordiente (sustancias químicas que son solubles en agua 
que facilita la unión del tinte y la fibra. El color obtenido no solo dependerá de la fuente, sino también del mordiente y el elemento a teñir (Boucherie, 2014). Las artesanas de Chincheros utilizan, hasta la actualidad, el mordiente de origen mineral, siendo el alumbre el más utilizado en las zonas Alto Andinas. En el proceso de tinción, Cornejo (1987) nos explica que en el caso del uso de las raíces, estas deben ser secadas y molidas antes de someterlas a la cocción. Con respecto a las cualidades y beneficios del uso de tintes naturales, los autores Cornejo (1987), Jaramillo (1988), Quintriqueo, Gutiérrez y Contreras (2012) y Boucheri (2014) comparten que el conocimiento de uso en comunidades conlleva al fortalecimiento de su identidad, reflejado en sus formas de trabajo y el valor cultural.

Frente a la creciente preocupación por la preservación ecológica y cultural de los pueblos originarios, es importante promover el interés por revivir el uso de tintes naturales.

\section{CONCLUSIONES}

Los datos que arrojaron las encuestas evidencian que la mayor parte de las familias que fabrican textiles y artesanías sigue persistiendo en las prácticas de tintes naturales, de generación en generación.

Es importante reconocer los beneficios de los tintes naturales a comparación de tintes artificiales. Debido a que en esta investigación, han obtenido mayor porcentaje de aceptación por parte de los artesanos en cuanto a calidad, tonalidad y gama de colores extraídos de plantas de la misma zona.

El uso de los tintes naturales ha demostrado tener mayor demanda en el distrito de Chincheros, por su calidad y en especial al fortalecimiento de su identidad cultural, esto reflejado a través de la conservación de las técnicas empleadas para la obtención del tinte y las formas de fijación sobre los textiles y objetos artesanales.

\section{REFERENCIAS}

Boucherie, N. (2014). La couleur dans la civilisation nasca: production tinctoriale et picturale, Lyon (tesis de doctorado). Université Lumière Lyon 2. Francia.

Cedano, M. (1994). Teñido con Hongos. Folleto instructivo, Universidad de Guadalajara, Instituto de Botánica, México.

Crespi, M. y Huertas J. (1987) Industria Textil: ¿Depuración Biológica o Fisicoquímica? Bol. Intextrar (92), 75-90.

Cornejo, Z. M. (1987). Características textiles del contexto de enterramiento La Horca Camaná (tesis de maestría). Universidad Católica Santa María, Arequipa.

Ivic, M. y Berger, M. (2008). El arte sale de las casas. Los tintes naturales en San Juan la Laguna Solola. Ciencia y Técnica Maya. Fundación Solar. Guatemala

Jaramillo, H. (1988). Textiles y Tintes. Ecuador: Centro Interamericano de Artesanías y Artes Populares (CIDAP).

Korovkin, T. (2002) Comunidades Indígenas, Economía del Mercado y Democracia en los Andes Ecuatorianos. Quito, Ecuador: AbyaYala.

Quintriqueo, S., Gutiérrez, M. y Contreras, A. (2012). Conocimientos sobre colorantes vegetales. Contenidos para la educación intercultural en ciencias. Perfiles Educativos, XXXIV(138), $108-123$

Sing, O. (1997). Colorantes naturales. Perú: Fondo Editorial, Pontificia Universidad Católica de $\mathrm{P}$ e r ú. R e c u p e r a d o d e : http://revistas.pucp.edu.pe/index.php/quimica /article/view/7562

Zumbuhl, H. (1979). Tintes naturales para lana de oveja. Huancayo, Perú. Karmaq maki, Sepas. 